\title{
Cross-Modal Collaborative Information Seeking (CCIS): An Exploratory Study
}

\author{
Dena Al-Thani, Tony Stockman, Anastasios Tombros \\ School of Electronic Engineering and Computer Science \\ Queen Mary University of London \\ Mile End road, London, E1 4NA, UK \\ \{dena.al-thani,tony,stockman,tassos\}@eecs.qmul.ac.uk
}

\begin{abstract}
Most previous studies of users with visual impairments access the web have focused solely on human-web interaction. This study explores the under investigated area of cross-modal collaborative information seeking (CCIS), that is the challenges and opportunities that exist in supporting visually impaired users to take an effective part in collaborative web search tasks with sighted peers. We observed behaviour patterns that occurred in CCIS activities between 8 pairs of visually impaired and sighted users in both co-located and distributed settings. Our findings showed the influence of the different interaction modalities employed, as well as differences due to whether pairs were working together co-located or distributed from one another. The effects of these factors were most clearly seen in the way pairs opted to divide the labour involved in search tasks, and the way in which they provided and used awareness information. Asymmetric division of labour strategies were employed to try to overcome the challenges imposed by accessibility issues and the use of different interaction modalities. We also observed that participants expended unnecessary effort in supplying more awareness information than was needed in an attempt to compensate for the absence of a tool to support cross-modal awareness information exchange.
\end{abstract}

Collaborative Information Seeking; cross-modal Interaction; accessibility; web search

\section{INTRODUCTION}

Activities that involve a group of people performing a search task with a shared information need or goal is referred to as Collaborative information seeking (CIS) (Morris, 2007). Research in the field of CIS has focused on providing solutions and frameworks to support CIS processes (Golovchinsky et al. 2012). The goal has been to enhance team productivity while performing a search task by allowing collaborators to search relevant information more effectively, avoiding redundant work and providing explicit support for collaborative processes. The work in this field to date, has always assumed that the collaborators engaged in CIS are using the same access modality, i.e. the visual modality. The focus on this modality has meant that $\mathrm{CIS}$ research has had limited value to people involved in CIS using other modes of interaction such as audio and/or haptic. For instance, visually impaired (VI) students and employees who use screen readers or Braille displays may often have to collaborate with their sighted peers to perform CIS tasks, in educational settings to complete group work or in employment as part of a collaborative information gathering effort.

Given that VI web searchers face a number of challenges due to the design of current screenreaders (Stockman and Metatla, 2008; Sahib et al., 2012); engaging in collaborative web search activities with sighted peers on reasonably equal terms is likely to be challenging. To date very little is known about the web search behaviour of $\mathrm{VI}$ users. The study by Sahib et al. (2012) highlighted the differences in individual information seeking (IS) behaviour between VI and sighted individuals and identified opportunities to improve support for individual web search by VI users. While the study by Sahib et al. (2012) is the only study of which we are aware with a specific focus on the accessibility of IS, as opposed to more general web-based interaction, still less is known about VI CIS behaviour, therefore this study focuses on understanding $\mathrm{VI}$ users involvement in $\mathrm{CIS}$ activities. The project overall has the primary goal of improving the accessibility of collaborative, Cross-modal Information Seeking (CCIS).

This paper presents the results of an exploratory study conducted to investigate CIS behaviour where the collaborators employ different modes of interaction for web search and related Computer Supported Collaborative Work (CSCW) activities. The study attempts to identify the challenges that occur in CCIS. A more detailed discussion of the research questions we aim to address is provided in section 3 .

\section{RELATED LITERATURE}

In order to study a cross-modal collaborative process it is important to analyse underlying 
activities and challenges that arise as part of the process. Therefore, this section will start by reviewing the relevant research from mainstream CIS. This is followed by covering the rather limited research in the field of cross-modal collaborative interaction.

\subsection{Collaborative Information Seeking (CIS)}

Morris (2008) surveyed the collaborative information seeking and information gathering habits of 204 technology workers. As a result of her study she recommended that in order to have an effective collaborative web search an interface should support awareness, division of labour and persistence.

The issue of awareness has been highlighted as early as the introduction of CSCW in the mid-80s. (Grief, 1988) A definition by Dourish and Bellotti (1992) describes awareness as "an understanding of the activities of others, which provides a context for your own activity". Research efforts in the field of CIS looked into either providing awareness in all stages of information seeking or focusing in providing awareness at different stages of the CIS process. These stages include query formation and results exploration. Systems that support CIS provided collaborators with the means of sharing queries and keywords entered, documents retrieved and comments within the group (Morris et al., 2010 and Paul and Morris, 2009). Results awareness is also explored, in which members can view the results of previously conducted searches or view search results of other members within the group. Microsoft's SearchTogether (Morris and Horviz, 2007a), a browser plugin designed to facilitate synchronous and asynchronous remote searching, provides a means of raising results awareness between collaborators

A number of division of labour strategies have been discussed in the CIS literature. These strategies are recognized and implemented according to the search task, the availability of resources and the role and expertise of the collaborators. The survey by Morris (2008) revealed two types of strategies: 'divide and conquer' and 'brute force'. The first strategy involves users working together explicitly throughout the search activity though the sub-tasks may be performed separately. The 'brute force' strategy, on the other hand, involves users not collaborating at all during the task but performing the entire process separately. Furthermore, it was observed that labour can also be divided asymmetrically based on collaborator role and expertise (Morris et al., 2008; Pickens et al. ,2008) and on the availability of technology resources (Amershi and Morris, 2008).

Apart from the features of CIS, Golovchinsky et al. (2009) proposed a taxonomy of CIS collaboration in which they introduced four dimensions of collaboration. These dimensions are: (1) Concurrency: synchronous and asynchronous (2) Location: co-located and distributed. (3) Intent: Explicit and implicit and (4) Degree of which CIS is mediated: user interface level and retrieval algorithm level. Moreover a recent publication by Morris et al. (2008) has stressed that the role of the collaborators and the task performed should also be considered essential dimensions of CIS.

\subsection{Cross-Modal interaction on the web}

Research in the field of cross-modal interfaces is relatively scarce. Nevertheless studies examining the collaborative web use between sighted and visually impaired users has highlighted that the serial nature of the synthesized speech imposes many challenges on collaborative use (Murphy et. al, 2008; Stockman and Metatla, 2008). These challenges include the lack of direct support for conveying web page structure, spatial organisation and context to $\mathrm{VI}$ users. These problems are exacerbated by the absence of an overview and the short-term memory overload that screenreaders can impose on their users. These barriers can enforce a sense of frustration on the $\mathrm{VI}$ when collaborating with their sighted colleagues (Lazar et al, 2006).

Murphy et al. (2007) studied the provision of audio and haptic feedback to users in an attempt to eliminate the differences in VI and sighted users' mental models of spatial navigation within a webpage. They developed and evaluated a multimodal browser that conveys spatial location using both auditory cues and a force feedback mouse. The results of their evaluation showed that the deployment of auditory cues can improve a VI user's cognition of spatial location, thus assisting the $\mathrm{VI}$ in developing a mental model of the page layout that is consistent with the sighted user's mental model.

Furthermore, Stockman and Metatla (2008) demonstrated the differences between the mental models of sighted and VI web users. They proposed a taxonomy of issues in cross-modal web-based collaboration and an agenda for how non-speech sound might be used to address some of these issues.

\section{RESEARCH QUESTIONS}

This observational study aims to identify and understand the challenges faced by VI and sighted users while performing CCIS tasks, as well as the strategies and techniques used to overcome these challenges. It looks into the effect of cross-modality interaction on the different aspects of CIS identified in the literature, particularly awareness and division of labour (Morris, 2007). We identified the following specific research questions: 
Q1: Does the presence of different modalities impose challenges on the CIS activity?

Previous research on individual VI user's IS behaviour has highlighted that most challenges are encountered during the results exploration phase (Sahib et al., 2012). Examining large search result sets using a screen reader can be a lengthy process, due to the issues that arise from the sequential nature of speech (Murphy et. al, 2008; Stockman and Metatla, 2008). Therefore this question aims to identify the affect that the presence of two modalities has on group performance and techniques employed to aid the process.

Q2: What mechanisms were used to facilitate awareness? And, relatedly,

Q3: How often are the participants aware of each other during the collaborative search task?

Literature on CSCW has always considered awareness as a fundamental aspect in interfaces that support collaboration. Providing an appropriate level of awareness can certainly enhance the CIS process (Shah and Marchionini, 2010). The current study looks into strategies employed to achieve group awareness. It investigates the methods being used especially in the absence of common access modalities, where it is no longer possible for users to share displays to be aware of one another's progress.

Q4: What strategies are used for the division of labour and how effective were these strategies?

The question examines division of labour strategies that emerge in the presence of two different modalities and the reasons behind employing these strategies. It also looks into the amount of labour completed by each collaborating pair in the study and issues that influenced the division of labour in the different pairs.

\section{METHODS}

The study involved the observation of 8 pairs of participants, each pair comprising a sighted and visually impaired searcher working together to gather information online for fictitious overseas trips. The study involved gathering information for two travel planning tasks, each set in a different country. For one task the participants were colocated and for the other they were situated remotely. This section starts by describing the participants, the set-up, the task and the procedure. It then introduces the data coding scheme employed.

\subsection{Participants}

The 16 participants were recruited in pairs; they were contacted through mailing lists. Details of the participants and the technologies they used are given in Table 1 below. Two pairs were colleagues who had been working together for more than two years. The other six pairs did not know each other. Three VI users used headphones, while the other five used speakers. All the VI users used the speech-only version of the Jaws screen-reader.

\begin{tabular}{|c|c|c|}
\hline & $\begin{array}{l}\text { Visually Impaired } \\
\text { Participants }\end{array}$ & $\begin{array}{c}\text { Sighted } \\
\text { Participants }\end{array}$ \\
\hline Age & $\begin{array}{l}1(21-29), 3(30-39) \\
3(40-49), 1(50-59)\end{array}$ & $\begin{array}{l}5(21-29), 2(40-49) \\
1(30-39)\end{array}$ \\
\hline Gender & 4 Male 4 Female & 5 Male, 3 Female \\
\hline $\begin{array}{l}\text { Browser } \\
\text { Used }\end{array}$ & $8 \mathrm{IE}$ & $\begin{array}{l}4 \text { IE, } 2 \text { Firefox } \\
1 \text { Safari, } \\
1 \text { Chrome } \\
\end{array}$ \\
\hline $\begin{array}{l}\text { Frequency } \\
\text { of CIS } \\
\text { Activity }\end{array}$ & $\begin{array}{l}\text { (2) Once a week } \\
\text { (1) once a month } \\
\text { (5) Once in the past } \\
\text { six months }\end{array}$ & $\begin{array}{l}\text { (2) Weekly } \\
\text { (2) once a month } \\
\text { (3) Once in the } \\
\text { past six months. } \\
\text { (1)Never }\end{array}$ \\
\hline
\end{tabular}

Table 1: Demographic and technology information about participants

\subsection{Set-up}

The intention of this study is to observe participants in real world settings; and so participants performed the collaborative search tasks in the VI participants' workplace. They were asked to use their own PCs and the web browser and search engines they normally use. In the distributed condition, participants were seated in remote locations and told that they could use one or more of the following methods to communicate: email, instant messaging, shared documents, or any tool they found suitable. While in the co-located condition, participants were seated in the same room and asked to communicate verbally though they were free to use additional methods. The sessions were all videotaped upon the approval of the participants and both participants' screens were captured using screen recording software. The VI participants' screens were captured using a video camera, as we noticed in a pilot of the experiment that screen recording software can slow down screen readers. Additionally the principal researcher made notes of observations during the sessions.

\subsection{Task}

Bruce et al. (2003) affirmed that the nature of the task in terms of its structure and context is an important factor that can influence collaborative behaviour. Surveys revealed that simple information look-ups and fact finding tasks do not benefit from CIS activity and that an exploratory search task is likely to benefit from CIS (London, 1995; Morris and Horvitz, 2007b). Therefore, the participants were asked to work collaboratively to organize a business trip to the United States (US) (in the co-located condition) and a holiday trip to 
Australia (in the distributed condition). They were given dates of engagements in different cities which were balanced across the two tasks. They were asked to organize the travel, accommodation and activities in these cities. In advance of each study we made sure that participants had not visited the cities before.

\subsection{Procedure}

Before performing the task participants were briefed about the purpose of the study and their given tasks. In the beginning of each session, participants were asked to fill a pre-study questionnaire which collected data such as age and gender as well as about the technologies they use for this type of task and their level of experience with web searching. Task order was balanced across the pairs of participants. In each task, the users were stopped after 35 minutes; they were not informed of this in advance as we were not interested in examining the influence of time pressure in this study. Following task completion, a brief, semi-structured interview was conducted. This helped in discussing the issues and challenges observed. Participants were also asked about other issues they encounter when performing similar activities.

\subsection{Data collection}

Participants' demographic and technology use information was captured through the preexperiment questionnaire. During the tasks the main source of data was the video recordings of the interaction between partners and their interactions with the search engines. All recordings were then transcribed and analysed to identify emerging patterns of behaviour. We used the Open and Axial coding phases of grounded theory (Strauss and Corbin, 1998). The coding scheme captures three main aspects: (1) The process (2) Information exchanged and (3) The purpose of the received information.

"The process" coding schema includes indicators of sub-tasks completion, and occurrences of accessibility issues. In terms sub-tasks completion, the given tasks in this study were quite structured; all pairs broke down the task into sub-tasks in a similar way. Each activity or accommodation or flight booking is considered as a sub-task. About 13 pieces of information are needed to be retrieved and put together. We used this coding approach to measure the number of sub-tasks accomplished across all pairs and the number of sub-tasks accomplished together. Though this criteria is task specific, it can help in estimating the amount of work completed by each participant. Additionally, we coded for the different accessibility issues encountered by participants during the study.
The "information exchange" coding scheme categories were identified during the coding. However, for the information that facilitates workplace awareness we have benefited from the framework developed by Gutwin and Greenberg (2002) which contains a set of elements relating to workplace awareness. We have refined these elements in order that they fit the CIS process. The coding scheme involved the following categories:

1. Information to facilitate the division of labour, which can be further categorized to: Initial division of tasks and reviewing outstanding tasks.

2. Information related to the problem at hand.

3. Information to facilitate cross-modal collaboration: It can be further categorised to request of assistant by the $\mathrm{VI}$ participant in relation to web accessibility issues or to difficulties in viewing a large amount of information (e.g. large search result set).

4. Information to facilitate workplace awareness: This category includes information of the status of the task. This information can either be supplied or requested by a participant.

The "The purpose of the received information" coding scheme, refers to the use of the received information by the participant. Four categories were developed. They are as follows:

1. Category A: Information that is filed away by the receiver. This is information that is not immediately needed.

2. Category B: Information that is needed but not critical. The receiver may use it in their next task but it isn't critical to completing the task.

3. Category C: The receiver absolutely needs it for their next task. The receiver will not be able to complete a given task without it

4. Category D: Information to avoid duplication of effort.

\section{RESULTS}

\subsection{Task completion}

The average number of sub-tasks completed in the co-located condition $(9.5, \mathrm{SD}=2.3)$ was higher than the average number of sub-tasks completed in the distributed condition (8.28, SD=2.7). Table 2 shows the average number of sub-tasks completed by each pair and sub-tasks completed together. Two factors were highlighted as the reason behind completing a task together. One of these reasons is related to needing to look at search results together and collaboratively make sense of the retrieved information. The other reason was that some websites were inaccessible and it was impossible for the VI partner to complete the task individually. 


\begin{tabular}{|l|l|l|}
\hline & \multicolumn{1}{|c|}{$\begin{array}{c}\text { Co-located } \\
\text { condition }\end{array}$} & $\begin{array}{c}\text { Distributed } \\
\text { condition }\end{array}$ \\
\hline $\begin{array}{l}\text { by the sighted } \\
\text { partner }\end{array}$ & $4.6(\mathrm{SD}=1.6)$ & $4.5(\mathrm{SD}=1.3)$ \\
\hline $\begin{array}{l}\text { by the VI } \\
\text { partner }\end{array}$ & $3.6(\mathrm{SD}=1.8)$ & $3.5(\mathrm{SD}=1.6)$ \\
\hline Together & $2.5(\mathrm{SD}=1.29)$ & $0.25(\mathrm{SD}=0.5)$ \\
\hline
\end{tabular}

Table 2: Average number sub-Tasks completed

\subsection{Challenges in relation to cross-modal collaboration}

The presence of two modalities impacted the way participants interacted during the IS task and influenced the division of labour in each condition. In the co-located condition it encouraged stages to be undertaken collaboratively, whilst this was not the case in the distributed condition.

In the co-located condition, VI users thought it might be quicker if they asked for assistance from their sighted partner. VI participants who had used booking websites or websites that contained inaccessible components before said that they delegated these tasks to their sighted partner to gain more time to complete other tasks.

In the distributed setting, when dividing the labour in the beginning of the study, usually visually impaired users delegated to their sighted partner sub-tasks that they know, from experience, it might take them some time to complete. Therefore, subtasks including hotel and flight booking that requires use of web sites that have inaccessible web components and/or require the VI user to spend time finding workarounds for accessibility issues. However, when VI users encountered an accessibility issue in the distributed condition, they tended to keep trying to complete the task, trying an average of 3 websites before involving their sighted partner to assist them.

In the observed sessions, a sum of 22 instances were recorded where the $\mathrm{VI}$ partner asked for assistance from their sighted partner in the colocated setting. $64 \%$ of these cases were accessibility issues while $36 \%$ of them were related to navigating large result sets. Only three requests for assistance were recorded in the distributed condition.

The majority of accessibility issues occurred while accessing hotel and flight booking websites. One participant described it by saying "It is a very very very frustrating process". Another participant commented during the experiment that "most of the websites don't work with screen-readers. I went to three websites and all of them don't work with JAWS because of accessibility issues". In the interviews, most of the VI users expressed frustration during interaction with websites that contained rich internet components such as Java Script, Flash videos and dynamic content. However, 2 VI participants stated that with experience they learned that there is usually a way around these issues but they preferred to request the assistance of their partner for the sake of saving time and effort to complete more tasks.

Navigating and searching websites with a large amount of information or links was highlighted as a challenge, as VI participants sought the support of their partner to navigate and look for required information. Though the majority used heading to heading navigation, an average of 2 requests for assistance was recorded per experiment in the colocated condition. One participant referred to the experience as a "struggle." He added; "unless I am familiar with the website, I will have to go through the whole website to understand the logic behind its structure". In the post-experiment interviews, when we asked the participants about the reason behind requesting for assistant when viewing search results, they stressed that they see it as a quicker way to gain insight about the information and so complete the given task.

\subsection{Information exchange between pairs}

In the co-located condition, collaborating partners exchanged awareness information verbally. As soon as the pair started performing the given task, they started to exchange information about the task and its status. In the distributed condition, five out of eight pairs preferred using emails, while the other three pairs chose to use instant messaging. They usually notify each other when they completed a sub-task and started another one. They also asked their partner about their status. In other words, they either supply or request information. Unlike verbal communication, in the distributed studies, an email can contain two or more categories of the data coding mention in the "information exchange" coding scheme. Below we present an excerpt of two conversations which are taken from each condition that show an example of instances of information exchange between participants, in which SP refers to the sighted participant and VP referees to the VI participant.

Co-located condition:

Dialogue 1:

VP: "Ok, flight is booked" (Category: Supplied, participant is describing what he just did)

Dialogue 2:

SP: "What you are doing?" (Category: Requested)

Distributed Condition:

Email exchange:

SP: Hi, I am now looking at Sidney dining (Category: Supplied, participant is describing what he will be doing next). Did you find the flight? (Category: Requested, participant is asking if a sub-task is completed) 
Instant messaging:

VP: Good. I found a tuxedo rental (Category: Supplied, participant describing what has just been done). I will now look for something to do in Melbourne on our free day.(Category: supplied, participant describing what he will do next)

Table 3 below summarizes the percentages of the number of occurrences of instances in the identified categories of coding. According to the categories identified, the highest proportion of information exchange is related to workplace awareness supplied information, while the proportion of requested information is low in both conditions. The cases where the participates supply their partners with information about "what they are doing" or "what they just did" has recorded the highest in both conditions.

\begin{tabular}{|l|l|l|}
\hline & $\begin{array}{l}\text { co-located } \\
\text { condition }\end{array}$ & $\begin{array}{l}\text { distributed } \\
\text { condition }\end{array}$ \\
\hline Division of labour & $23 \%$ & $16 \%$ \\
\hline $\begin{array}{l}\text { Request for assistant } \\
\text { in web accessibility } \\
\text { issue }\end{array}$ & $12 \%$ & $2 \%$ \\
\hline $\begin{array}{l}\text { Request for viewing } \\
\text { large amount of } \\
\text { information }\end{array}$ & $2 \%$ & $0 \%$ \\
\hline Supplied Information & $42 \%$ & $64 \%$ \\
\hline Requested Information & $13 \%$ & $10 \%$ \\
\hline $\begin{array}{l}\text { Task related } \\
\text { discussion }\end{array}$ & $11 \%$ & $8 \%$ \\
\hline
\end{tabular}

Table 3: Percentages of the number of occurrences of instance of each information exchange category

The information related to division of labour, is further categorised into two subcategories. In the co-located condition, $75 \%$ of the information exchanged in this category related to the initial division of tasks, which happens in the start of the session. This exchange of information involved participants reviewing the task and discussing the division of labour by assigning tasks to one another. While $25 \%$ of the information exchanged in this category is related to "reviewing the outstanding task" category. Interestingly, this subcategory was not found in the distributed condition which indicates that remote collaborators tend to divide the labour in the very beginning of the task and don't discuss the status of the task later.

In relation to information exchanged that is related to cross-modality in the co-located setting $84 \%$ of the cases related to this category involved accessibility issues. They either contain a request for assistance or a notification that the participants are currently facing an accessibility issue that may delay task completion. $16 \%$ of information exchanges in this category are related to viewing large amounts of information, in which a VI participant may request assistance to review a large amount of data. However, this was not the case in the distributed setting, where all the cases recorded were accessibility issues and no instances of request for assistance to view large amount of data were recorded, presumably because this would be harder, though not impossible, in the distributed condition.

\subsection{The usage of the received information}

When looking at the usage of the received information and what the receiver does with it, as shown in Table 4 the major difference between the two conditions was in the amount of critical information exchanged. The amount of critical information was extremely low in the distributed conditions in comparison to the co-located condition. This is related to the strategy collaborators used to divide labour. The task is divided in a way that the sub-tasks are less dependent on each other; hence less critical information is exchanged. The amount of information to avoid duplication is slightly higher in a distributed setting. When the collaborators work remotely they tend to update each other more often.

\begin{tabular}{|l|c|c|c|c|}
\hline & A & B & C & D \\
\hline $\begin{array}{l}\text { Co-located } \\
\text { condition }\end{array}$ & $27 \%$ & $30 \%$ & $20 \%$ & $22 \%$ \\
\hline $\begin{array}{l}\text { Distributed } \\
\text { condition }\end{array}$ & $27 \%$ & $36 \%$ & $9 \%$ & $26 \%$ \\
\hline
\end{tabular}

Table 4: Percentages of each category in "the usage of the received information"

Analysing the figures has shown a relationship between the type of information exchanged and participants' performance in the tasks. Figure 1 shows the percentage of the average number of information exchanged by category in studies with the highest performance and studies with the lowest performance from each condition. The performance of each study is measured by the amount of sub-tasks completed by the pair in the session.

When looking into the studies were partners performed very well in the co-located condition, in the top three studies the average number of information exchanges related to information that is needed but not critical (category B) is high. In these top performing pairs, the average number of critical information exchanges (Category $\mathrm{C}$ ) was at the lowest. In the top three distributed studies, the amount of information exchanged to avoid duplication of effort (Category D) is highest. Additionally, the amount of information exchange for needed but not critical information (Category B) was also fairly high. Yet, the amount of critical information (Category $\mathrm{C}$ ) is the lowest. 


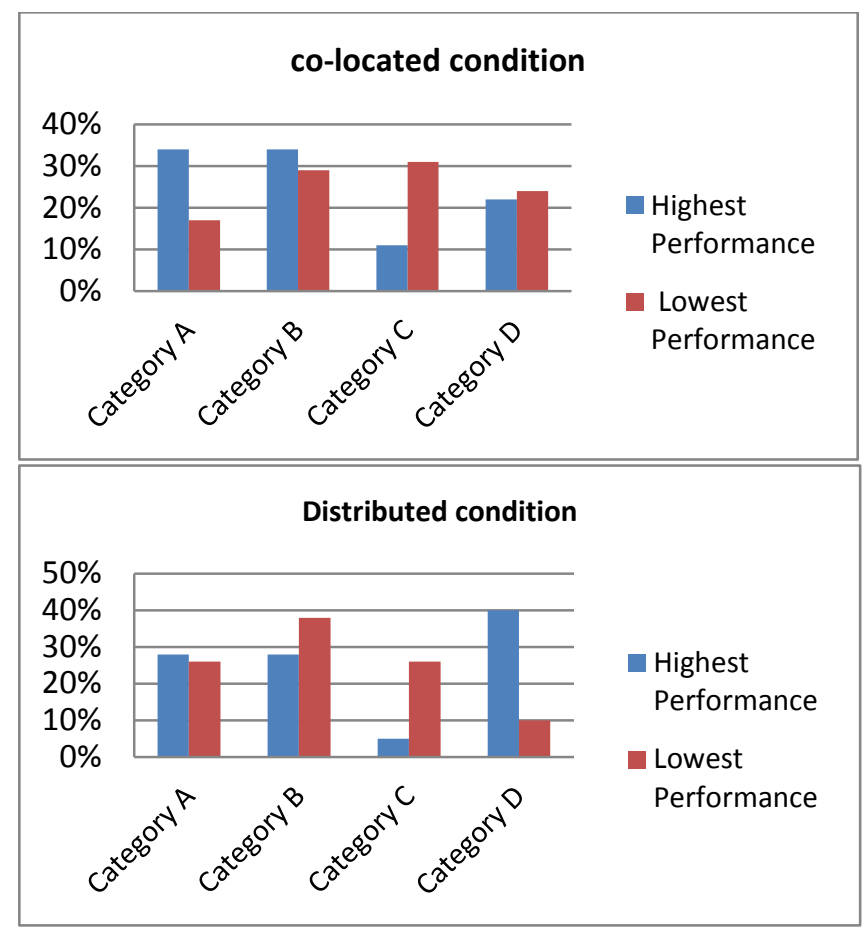

Figure 1 the percentage of each category in the studies with highest performance and studies with lowest performance in each condition

When looking into the studies where partners' performance is relatively low in the distributed condition the amount of information exchange to avoid duplication (Category D) is the lowest. In these studies the amount of needed but not critical information is the highest (Category B). It is also noted that the amount of critical shared information (Category $\mathrm{C}$ ) is remarkably high in comparison to the pairs with higher performance. Similarly, in the co-located studies where the performance was low (Category $\mathrm{C}$ ) was high and (Category $\mathrm{A}$ ) is at the lowest.

\section{DISCUSSION AND ANALYSIS}

\subsection{Awareness}

Shah and Marchionini (2010) asserted that in CIS activities the level of group and workplace awareness can play a significant role in the overall performance of the collaborators; and supporting a sufficient level of awareness can have more impact on the time efficiency than any other feature of the CIS. In the co-located setting the main method to implement awareness was verbal communication. However, in many instances sighted partners were observed looking at the screen of their VI partner to be able to know their partner's status. While in the distributed condition the only method to implement awareness was through email or instant messaging. Two types of behaviour were observed to facilitate group and workplace awareness. The first one is the awareness of a partner's activity at a given time. Participants need to know their partners' actions at a certain point in time. In this example the participant either notifies his/her partner about the task they are working on or asks his/her partner about the task being performed. The second type is awareness of the overall progress on the task. This type of behaviour to support workplace awareness usually happens when participants discuss their work. At discussion stages and mainly toward the end of the task participants frequently review the completed subtasks and identify the remaining ones.

To facilitate awareness of the activities of their partner while performing the task, participants supplied their partner with information about the activity they were performing. The information provided can be classified into three forms; information about the sub-task that has just been started, the sub-task currently being performed, and the sub-task that has just been completed at a given time. The information received can be classified into four forms; information not needed for the current sub-task, information needed but not critical to the current sub-task, information critical to the current sub-task and information to avoid duplication of effort.

The percentage of information provided by the participants was higher than the information requested. The amount of information supplied but considered not relevant for the current sub-task or considered not critical by the receiver of this information was relatively high in both conditions. Even though the sub-tasks are quite structured and the majority of these sub-tasks are not dependent on each other, participants constantly kept supplying each other with information to enrich workplace awareness in the absence of a tool that supported awareness in both conditions. In fact, with pairs with a higher performance in the colocated condition, more than $60 \%$ of the information exchanged was information that is not critical but might be used later (Categories $A$ and $B$ ).

The amount of information to avoid duplication (Category D) was much higher in the distributed condition. Moreover, it was observed that when more information to avoid duplication was available, the higher was the performance. This indicates that making this type of information available between distributed collaborators could itself enhance their ability to complete tasks in a timely manner. This was not the case in the studies performed in the co-located setting, as the studies with lowest and highest performance reported the same amount of information exchanged relating to duplication of effort.

Facilitating awareness of the overall progress on the task was observed on average two to three times during the co-located sessions and was typically included within the division of labour discussions. This type of awareness information was not observed in the distributed sessions. The 
frequency with which it occurs is highly dependent on the number of sub-tasks accomplished and the division of labour strategy applied. In this type of behaviour one or both pairs try to put together all the information found and then check for missing information.

\subsection{Division of labour}

The main purpose of the division of labour is to avoid unnecessary duplication of effort in joint activities and to enhance efficiency. It was observed that in the co-located setting, the pairs usually tend to employ the 'divide and conquer' strategy (Morris, 2008) to divide labour. In this strategy although the main task is divided into subtasks and assigned to each partner, the pair continue discussing the work together throughout the process. Whereas in the distributed setting it was observed that the collaborators used a 'brute force' strategy. (Morris, 2008), in which the participants work independently and only notify each other when a sub-task is completed.

In the co-located setting, discussion about the division of labour occurred at two levels. Firstly in the initial discussion and secondly as a result of one participant interrupting his/her partner in order to complete their current sub-task together. In the co-located setting, the pair worked together at many points throughout the process. Three reasons were identified for these collaborations:

1. When browsing websites with large volumes of data, VI users asked sighted partners to help them by scanning the page for specific information to speed the task.

2. When browsing websites with inaccessible components, VI users asked sighted participants to perform the task or assist them in performing it.

3. The third reason is related more to the context of the task. Participants tended to work together when choosing places to visit and discussing the choices available. In this type of task the participants worked together when exploring search results. Morris (2008) also emphasized this type of model where the type of task influenced the division of labour process.

In contrast, in the distributed setting, the discussion about the division of labour occurred only at the beginning of the task. The pair divided the work and started work independently. Therefore, critical information exchanges were at the lowest in the distributed condition, being $9 \%$ of the total information exchange, while it was about $20 \%$ in the co-located studies. Additionally, request for assistance were at the lowest in this condition. Similarly to the co-located condition, sighted users assisted VI users for the same reasons as 1 and 2 above, though VI partners were more reluctant to ask for this support in the distributed condition. Pickens et al. (2008) introduced the concept of asymmetric division of labour in their "gatherer and surveyor model", in which one partner would search for new information and the other partner would look through the large volume of search results. The strategies used by participants in our study were often of an asymmetric type.

It is clear that both the task and condition of the collaborative activity had a great influence on how labour was divided. Moreover, awareness and division of labour could not be looked at separately as the level of awareness has potential impact on the way labour is divided (Morris et al., 2004; McGookin and Brewster, 2007; Metlata, 2010). It was observed that in each of the strategies employed, the percentages of the types of information exchange differ. For instance, in the colocated setting the amount of critical information exchanged was higher as the task was divided in such a way that the sub-tasks were more dependent on each other.

Additionally, both the type of awareness information exchanged and strategy employed to divide labour highly impacted the performance of pairs. The more independently individuals performed sub-tasks on their own, the less was the amount of critical information exchanged and the higher the performance was in both co-located and distributed conditions. Further, the more information that was available to avoid duplication, the higher was the performance in the distributed condition.

\section{CONCLUSION}

This paper presents the findings of an observational study of CIS processes in the presence of two different modalities and in two different conditions. The Findings showed that there is a clear influence of the different modalities and conditions on both individual and collaborative work. Awareness played an important role in both the strategies chosen and the degree to which labour was divided successfully and efficiently.

The study yields interesting results in terms of the challenges posed by CCIS that can be a focus for improvement. Facilitating awareness of CIS activity in a cross-modal context can certainly support the CCIS process. Very few studies have explored supporting awareness in accessible cross-modal collaboration (e.g. Winberg and Brewster, 2004; McGookin and Brewster, 2007, Metatla, 2010). Primarily, these studies examined conveying information about group members' activities using sound. limproving the awareness of partners' IS 
activities in a cross-modal context appears to be a fruitful way forward. While awareness in both mainstream CIS (Shah and Marchionini) and crossmodal interaction (e.g. (McGookin and Brewster, 2007) and (Metlata et al., 2012)) is gaining more interest recently, it has not been explored in the context of information seeking.

Furthermore, it was clear that underlying individual IS issues faced by VI users have enforced an asymmetric division of labour among collaborators. Addressing these issues can certainly affect the way labour is divided between collaborators and enhance the overall performance. Supporting the way VI users approach large volumes of search results can help in increasing the independence of the VI user. It will clearly bring about improvements in both individual and collaborative IS activities.

\section{REFERENCES}

Amershi, S., \& Morris, M. R. (2008). CoSearch: a system for co-located collaborative web search. Proceedings of the twenty-sixth annual SIGCHI conference on Human factors in computing systems, CHI '08, New York, NY, USA, 16471656.

Bruce, H., Fidel, R. Pejtersen, A.M., Dumais, S., Grudin, J. \& Poltrock, S. (2003). A comparison of the collaborative information retrieval (CIR) behaviors of two design teams. New Review of Information Behaviour Research, 4, 139-153.

Dourish, P., \& Bellotti, V. (1992). Awareness and coordination in shared workspaces. In Proceedings of the 1992 ACM conference on Computer-supported cooperative work, New York, NY, USA, 107-114

Golovchinsky, G., Dunnigan, A., \& Diriye, A. (2012). Designing a tool for exploratory information seeking. In Proceedings of the 2012 ACM annual conference extended abstracts on Human Factors in Computing Systems Extended Abstracts, 1799-1804.Austin, TX, USA, (pp. 1647-1656.

Golovchinsky, G., Pickens, J., \& Back, M. (2009). A taxonomy of collaboration in online information seeking. Proceedings of the 1st International Workshop on Collaborative Information in RetrievalJCDL '08, Pittsburgh, PA, USA

Greif, I. (1988). Computer-supported cooperative work: A book of readings. Morgan Kaufmann Pub.

Gutwin, C., \& Greenberg, S. (2002). A Descriptive Framework of Workspace Awareness for RealTime Groupware. Computer Supported Cooperative Work (CSCW), 11(3-4), 411-446.

Lazar, J., Feng, J., \& Allen, A. (2006). Determining the impact of computer frustration on the mood of blind users browsing the web. In Proceedings of the 8th international ACM SIGACCESS conference on Computers and accessibility, New York, NY, USA, 149-156.

London, S. (1995). Collaboration and community. Richmond, VA, Pew Partnership for Civic Change, University of Richmond. Retrieved from http://www.upperskeena.ca/storytellers/CCL\%20 research/ccl/themes/micromacro/collaboration.pdf

McGookin, D., \& Brewster, S. (2007). An initial investigation into non-visual computer supported collaboration. $\mathrm{CHI} 07$ extended abstracts on Human factors in computing systems, San Jose, CA, USA, 2573-2578.

Metatla, O., (2010). Collaborating Through Sounds: Audio-Only Interaction with Diagrams. Ph.D. thesis, Queen Mary University of London, London, UK.

Metatla O., Bryan-Kinns N., Stockman T., Martin F., (2012) Cross-modal Collaborative Interaction Between Visually-impaired and Sighted Users in the Workplace. Proceeding of International Conference of Auditory Display, ICAD'2012, Georgia Institute of Technology, Atlanta, GA, USA.

Morris, M. R. (2007). Collaborating alone and together: Investigating persistent and multi-user web search activities. In Proc. Int. ACM SIGIR Conf. on Research and Development in Information Retrieval (SIGIR'07). 23-27.

Morris, M. R. (2008). A survey of collaborative web search practices. Proceedings of the twentysixth annual SIGCHI conference on Human factors in computing systems, CHI '08, New York, NY, USA, 149-156.

Morris, M. R., \& Horvitz, E. (2007a). SearchTogether: an interface for collaborative web search. Proceedings of the 20th annual ACM symposium on User interface software and technology, Newport, RI, USA, 3-12.

Morris, M., \& Horvitz, E. (2007b). S3: Storable, Shareable Search. In C. Baranauskas, P. Palanque, J. Abascal, \& S. Barbosa (Eds.), Human-Computer Interaction - INTERACT 2007, Lecture Notes in Computer Science (Vol. 4662, pp. 120-123). Springer Berlin / Heidelberg. Retrieved from http://www.springerlink.com/content/bg77hn9626 071756/abstract/

Morris, M. R., Lombardo, J., \& Wigdor, D. (2010). WeSearch: supporting collaborative search and sensemaking on a tabletop display. Proceedings of the 2010 ACM conference on Computer supported cooperative work, CSCW '10, New York, NY, USA, 401-410. 
Morris, M. R., Morris, D., \& Winograd, T. (2004). Individual audio channels with single display groupware: effects on communication and task strategy. In Proceedings of the 2004 ACM conference on Computer supported cooperative work, New York, NY, USA, 242-251.

Morris, M.R., Paepcke, A., \& Winograd, T. (2006).TeamSearch: Comparing Techniques for Co-Present Collaborative Search of Digital Media. IEEE Tabletop 2006, 97-104.

Morris, M. R., Teevan, J., \& Bush, S. (2008). Enhancing collaborative web search with personalization: groupization, smart splitting, and group hit-highlighting. Proceedings of the 2008 ACM conference on Computer supported cooperative work, CSCW '08, New York, NY, USA, 481-484.

Morris, M. R., Teevan, J., \& Panovich, K. (2010). What do people ask their social networks, and why?: a survey study of status message q\&a behaviour. Proceedings of the 28th international conference on Human factors in computing systems, CHI '10, New York, NY, USA, 17391748.

Murphy, E., Kuber, R., McAllister, G., Strain, P., \& Yu, W. (2008). An empirical investigation into the difficulties experienced by visually impaired Internet users. Universal Access in the Information Society, 7(1), 79-91.

Murphy, E., Kuber, R., Strain, P., McAllister, G., \& Yu, W. (2007). Developing sounds for a multimodal interface: conveying spatial information to visually impaired web users. Proceedings of the 13th International Conference on Auditory Display (ICAD '07), 2629.

Paul, S. A., \& Morris, M. R. (2009). CoSense: enhancing sensemaking for collaborative web search. Proceedings of the 27th international conference on Human factors in computing systems, CHI '09,. New York, NY, USA, 17711780.

Pickens, J., Golovchinsky, G., Shah, C., Qvarfordt, P., \& Back, M. (2008). Algorithmic mediation for collaborative exploratory search. Proceedings of the 31 st annual international ACM SIGIR conference on Research and development in information retrieval, SIGIR '08, New York, NY, USA, 315-322.

Sahib, N. G., Tombros, A. and Stockman, T. (2012) A comparative analysis of the information seeking behavior of visually impaired and sighted searchers. Journal of the American Society for Information Science and Technology, 63(2): 377-391/
Shah, C., \& Marchionini, G. (2010). Awareness in collaborative information seeking. Journal of the American Society for Information Science and Technology, 61(10), 1970-1986.

Stockman, T., \& Metatla, O. (2008). The influence of screen-readers on web cognition. Proceedings of Accessible design in the digital world conference (ADDW 2008), York, UK.

Strauss, A. C. \& Corbin, J. M., (1998). Basics of Qualitative Research: Techniques and Procedures for Developing Grounded Theory. Beverley Hills, CA: Sage

Winberg, F., \& Bowers, J. (2004). Assembling the senses: towards the design of cooperative interfaces for visually impaired users. In Proceedings of the 2004 ACM conference on Computer supported cooperative work. New York 